status epilepticus is more sensitive than the MRI as a biomarker for the prediction of subsequent epilepsy.

Previous studies of the EEG in children with febrile seizures, some performed in the 1950s-60s and cited by the FEBSTAT investigators, also emphasize the frequency and significance of slow wave abnormalities. In addition, Lennox-Buchthal M (Ugeskr Laeger 1964 Feb 13;126:203-6) extended her research and performed serial EEGs on children with febrile seizures at varying time intervals after the first seizure as follows: on the third, fourth, or fifth day of admission, 10 days to 2 weeks after admission, and at 3, 6 , and 12 months and every year at follow-up visits. The EEGs showing slow frequencies at the third to fifth day after a febrile convulsion had a trace of slowing in some after 10 days to 2 weeks, but the record was normal after an interval of 3 months. Compared to the patients with normal initial EEGs, those with pronounced slow frequencies had a greater incidence of recurrence of febrile seizures and of paroxysmal abnormalities in subsequent records, but the differences in incidence were not significant. Perhaps the larger cohort of the FEBSTAT study will show a more robust correlation between slowing and subsequent epilepsy at follow-up. Repeated EEGs at intervals are advised in some febrile seizure patients with recurrent seizures or other complications so that delayed emergence of paroxysmal abnormalities and susceptibility to epilepsy with increasing age may be excluded.

\title{
CSF IN FEVER-ASSOCIATED STATUS EPILEPTICUS
}

Investigators at Children's Hospital of the King's Daughters, Eastern Virginia Medical School, Norfolk, VA; Albert Einstein College of Medicine, Bronx, NY; Columbia University, NY; and other centers on behalf of the FEBSTAT Study Team assessed CSF findings in $154(77 \%)$ of 200 patients with fever-associated status epilepticus (FSE). LP was performed at the discretion of the attending physicians, and 136 children had a nontraumatic LP $(<1000$ red blood cells; $116(96.2 \%)$ of the 136 had

$</=3$ white blood cells $/ \mathrm{mm}^{3}$ ). Likelihood of an LP performed in the ED was significantly higher in younger children, in those with the first FS, a longer median duration of FSE, febrile status epilepticus, and a focal FSE. Mean CSF protein level was $22 \mathrm{mg} / \mathrm{dL}$ (range, $8-137 \mathrm{mg} / \mathrm{dL}) ; 3$ (2.3\%) had a protein level $>60 \mathrm{mg} / \mathrm{dL}$. Mean CSF glucose level was $89.6 \mathrm{mg} / \mathrm{dL}$ (range, 46-201 mg/dL). Excess WBCs in the CSF should not be attributed to the seizure (Frank LM, Shinnar S, Hesdorffer DC, et al. Cerebrospinal fluid findings in children with fever-associated status epilepticus: Results of the Consequences of Prolonged Febrile Seizures (FEBSTAT) Study. J Pediatr 2012 Dec;161(6):11691171.e1). (Reprint requests: L. Matthew Frank MD. E-Mail: matthew.frank@chkd.org).

COMMENT. LP performed at the discretion of the attending physician confirms that the CSF is usually normal in children with FSE. Abnormal CSF results should not be attributed to the seizure and should prompt close investigation and treatment for suspected meningitis.

Clinical manifestations and complex seizures are the principal indications for lumbar puncture, not patient age, in a study of 100 consecutive febrile seizure patients treated in a tertiary hospital ED. (Millichap JJ et al. Pediatr Neurol 2008 Dec;39(6):3816). Eleven (78.6\%) patients undergoing LP had complex FS, 3 manifesting prolonged 
seizures and FSE, with durations of 43,45 , and 60 minutes. CSF findings were normal and bacterial cultures were negative. A child aged 3 months to 5 years who presents with a first or recurrent FS should be considered for LP if one or more of the following indications are present: neurologic signs of meningitis, systemic signs of toxicity, complex seizure with prolonged postictal obtundation of consciousness, or pretreatment with antibiotics. Complex FS alone is not an absolute indication for LP.

In a retrospective study at Children's Hospital Boston to assess the rate of acute bacterial meningitis among 526 children who present with their first complex febrile seizure, $2.7 \%$ had CSF pleocytosis and 3 patients $(0.9 \%)$ had acute bacterial meningitis. One appeared well clinically; of 2 with Streptococcus pneumoniae cultured from CSF, 1 was nonresponsive clinically, and the other had a bulging fontanel and apnea. (Kimia A, et al. Pediatrics $2010 \mathrm{Jul} ; 126(1): 62-9)$.

\section{THALAMOCORTICAL STRUCTURAL AND FUNCTIONAL CONNECTIVITY IN JUVENILE MYOCLONIC EPILEPSY}

Researchers at King's College, Institute of Psychiatry, London and other centers in the UK, US, and Germany discovered changes in an anterior thalamo-cortical bundle during tests of structural connectivity, as measured by diffusion tensor imaging, in a cohort of 28 subjects with juvenile myoclonic epilepsy. An alteration in task-modulated connectivity was detected in a region of frontal cortex connected to the thalamus via the same anatomical bundle, and overlapping with the supplementary motor area. In patients with active seizures, the degree of abnormal connectivity is related to disease severity in those with active seizures. These results point to abnormalities in a specific thalamocortical circuit, with reduced structural and task-induced functional connectivity that underlies this idiopathic epilepsy. (O'Muircheartaigh J, Vollmar C, Barker GJ, et al. Abnormal thalamocortical structural and functional connectivity in juvenile myoclonic epilepsy. Brain 2012 Dec;135(Pt 12):3635-44). (Response: Dr Mark P Richardson, Email: mark.richardson@kcl.ac.uk).

COMMENT. The characteristic generalized spike and wave discharges in the EEG of juvenile myoclonic epilepsy implicate thalamo-cortical interactions, and the discharges are most prominent in frontal regions. The functional and diffusion MRI and diffusion tensor imaging used above provide anatomic evidence for the role of the thalamus and a specific thalamo-cortical circuit dysfunction in JME. JME is a lifelong disorder and a structural cerebral defect may explain the necessity to continue treatment indefinitely. (Wandschneider B, et al. Frontal lobe function and structure in juvenile myoclonic epilepsy: a comprehensive review of neuropsychological and imaging data. Epilepsia 2012 Dec;53(12):2091-8).

\section{MUSCULAR DYSTROPHY-DYSTROGLYCANOPATHY AND EPILEPSY}

Investigators from the University of Catania, and other centers in Europe have identified a novel genetic glycosylation disorder, DPM2-CDG (part of the DPM synthase complex) in 3 infants with severe hypotonia, progressive muscle weakness and wasting, elevated CK, absent psychomotor development, intractable epilepsy with onset at 1 week 